\title{
Vascular opacification and leakage in X-linked (juvenile) retinoschisis
}

\author{
JAMES L. GREEN JR AND LEE M. JAMPOL \\ From the Department of Ophthalmology, University of Illinois Eye and Ear Infirmary, Chicago, USA
}

\begin{abstract}
SUMMARY A 22-year-old man had X-linked retinoschisis with extensive vascular sheathing in the posterior pole and dendritic opacified retinal vessels in the periphery. Detailed fluorescein angiographic studies were performed. In the far periphery areas of capillary nonperfusion were seen. Sluggish circulation was present in the majority of dendritic opacified vessels in the areas of schisis. Late staining of the disc and posterior arteries and veins was noted. Scattered intraretinal fluorescein leakage was present even in clinically nonschitic retina, which suggests that the vascular changes may precede the retinoschisis. The patient's half-brother also had X-linked retinoschisis, but the schisis and the vascular changes were much less prominent. Although the retinal vascular changes with X-linked retinoschisis are similar to those seen with the Favre-Goldmann syndrome, a differentiation of the two diseases can be made by family studies, the presence or absence of nyctalopia, examination of the macular areas, measurement of dark adaptation, and electroretinogram determinations.
\end{abstract}

Juvenile retinoschisis is an $\mathrm{X}$-linked recessive vitreoretinal dystrophy with a high degree of penetrance and variable expressivity (Deutman, 1977). Ophthalmoscopic findings include foveal changes (cystoid spaces with a bicycle-spoke pattern of radial striae), vascular sheathing, peripheral retinoschisis, vascularised veils in the vitreous, occasional vitreous haemorrhage, pigmentary changes in the fundus, and in some cases white dendritic arborescent structures in the peripheral retina. Previous fluorescein angiographic studies (Krause et al., 1970; Burns et al., 1971; Constantaras et al., 1972; Tasman, 1975; Harris and Yeung, 1976) of X-linked retinoschisis have concentrated primarily on changes in the posterior pole, while the vascular changes in the peripheral retina have received scant attention (Ewing and Ives, 1969; Ewing and Cullen, 1972; Tasman, 1975). We recently examined 2 brothers with X-linked retinoschisis. One had large areas of retinoschisis with unusually prominent dendritic structures (Cibis, 1965) in the retinal periphery and marked vascular sheathing in the posterior pole. The other had small areas of peripheral retinoschisis with early vascular opacification. Our findings on ophthalmoscopy and fluorescein angiography show

Address for reprints: Dr Lee M. Jampol, University of Illinois Eye and Ear Infirmary, $1855 \mathrm{~W}$ Taylor Street, Chicago, Il. 60612, USA that retinal vascular changes can be a prominent feature of the clinical picture of X-linked retinoschisis.

\section{Case reports}

\section{CASE 1}

A 22-year-old man of Mexican descent came to the Illinois Eye and Ear Infirmary in September 1977 with a complaint of bilateral progressive decrease in vision of about 10 years' duration. Previously his vision was said to have been normal, but no previous ophthalmological evaluations had been performed. The patient also complained of mild photophobia, but he specifically denied night blindness. His general medical health was excellent, and there was no family history of ophthalmological problems, except for one half-brother whose case is described below (Case 2). No other family members were available for examination.

Ophthalmological examination showed a best corrected visual acuity in the right eye of counting fingers $\left(+5.00 \mathrm{~S}+1.25 \mathrm{cx} 175^{\circ}\right)$, left eye $20 / 200$ $\left(+2 \cdot 25 \mathrm{~S}+3.25 \mathrm{cx} 170^{\circ}\right) ; 15^{\circ}$ of right exotropia were seen with an afferent pupillary defect on the right. Slit-lamp biomicroscopy of the anterior segment and applanation tonometry were normal. No cataracts were present. Ophthalmoscopy disclosed normal optic discs, but there was marked 
vascular sheathing in the posterior pole, the arteries being slightly more involved than the veins (Fig. 1). In the right eye the vascular sheathing continued inferiorly to a large area of retinoschisis (Fig. 2). The schisis involved the entire inferotemporal quadrant. The remainder of the fundus, including the posterior pole, had a recticular texture (Fig. 3). The macula showed a characteristic bicyclespoke appearance with coalescence of many of the cystoid spaces.

Examination of the fundus on the left showed 2 large highly elevated schisis cavities, 1 inferior and 1 superotemporal. The inferior cavity occupied most of the inferior periphery and extended from near the ora serrata to within 4 disc diameters of the optic nerve and macula. The reticular pattern found in the right eye was much less prominent and was present only at the posterior edges of the schisis cavities. The macular area showed cystoid changes but without the coalescence seen in the right eye (Fig. 4). A large inner-layer retinal hole was seen in the area of schisis in the left eye superotemporally (Fig. 5).

The periphery of both eyes in areas of retinoschisis showed a prominent dendritic pattern of whitish opacified vessels (Figs. 2 and 5). These vessels appeared to represent small arterioles and venules as well as capillaries. The changes were most pronounced in the midperiphery and were less prominent anterior to the equator and at the posterior margin of the schisis. A mild degree of vessel opacification was apparent, however, along the posterior margins of the schisis extending into apparently nonschitic retina (Fig. 6). The majority of opacified vessels were seen in the inner layer of schisis, but in some areas white vessels were noted in both layers of the schisis cavity (Fig. 5).

Dark adaptation testing showed a normal cone final threshold. The final rod threshold was raised $0.5 \log$ unit at $15^{\circ}$ superior to the fovea, testing with a $2^{\circ}$ white test target using the GoldmannWeekers dark adaptometer. Colour vision testing (Helve, 1972) with the Farnsworth-Munsell 100-hue test showed a blue-yellow defect in the left eye. The electroretinogram (ERG) was obtained by techniques previously described (Fishman et al., 1977). The photopic response in the left eye showed normal amplitudes and implicit times. The right eye amplitudes were minimally subnormal with normal implicit times. The scotopic response bilaterally produced a normal a-wave amplitude, while the b-wave amplitude was significantly reduced, giving a negative type response. A flicker response produced with red light at 30 cycles per second showed a subnormal response in the right eye and a normal response in the left eye. A flicker response pro- duced with a low-intensity blue stimulus showed moderately subnormal amplitudes in each eye, with the right eye being depressed more than the left.

Fluorescein angiography was performed in the macula and periphery of both eyes. Angiography confirmed that the white dendritic lesions were opacified retinal vessels (Figs. $7 a-7 c$ ). The circulation in the vessels was markedly delayed. Anterior to the vascular opacification areas of extensive capillary nonperfusion were apparent (Fig. 7c). A few of the opacified vessels were also not perfused, though the majority of them were patent. Posterior to the schitic areas patchy staining of vessel walls with some intraretinal leakage of fluorescein was present in some areas (Fig. 8). Views of the posterior pole showed no definite retinal leakage into the cystoid spaces in the maculae (Figs. 9a and 9b). Mild disruption of the pigment epithelium was apparent in the right macula with window defects visible in two areas. Other areas of pigment disruption were present near the disc in the right eye and in the periphery. The walls of large arteries and veins and the discs in both eyes stained with fluorescein in late views (Fig. 9b).

\section{CASE 2}

This patient is the 30-year-old half-brother of Case 1. They have the same mother but different fathers. The patient was asked to come in for ophthalmological examination, though he had no visual complaints. When queried, however, he did state that his right eye was 'not as good' as his left eye. He denied night blindness.

Ocular examination showed a best corrected visual acuity in the right eye of 20/200 $(+4.75 \mathrm{~S}$ $+2.00 \mathrm{cx} 150^{\circ}$ ), left eye $20 / 70 \dashv$ (plano $+1.50 \mathrm{cx}$ $70^{\circ}$ ). The extraocular muscle balance and the pupillary responses were normal. Slit-lamp biomicroscopy of the anterior segment and applanation tonometry showed nothing abnormal.

Fundus examination on the right disclosed a normal disc and a bicycle-spoke pattern of macular cystoid changes. No posterior vascular sheathing was noted. The peripheral retina showed areas of mild pigment disruption and other areas with a peculiar sheen. No elevated schisis was present, but 2 small (1 disc diameter) areas of opacified capillaries were found, 1 inferior at 6 o'clock and 1 superotemporal at 9.30 o'clock.

Ophthalmoscopic examination of the left eye again showed the characteristic macular cystoid changes. The remainder of the retina was normal with the exception of a temporal semi-circular ridge of tissue that represented the remnant of a schisis cavity (Fig. 10). A small area of opacified vessels was seen at the inferior border of this ridge. A 


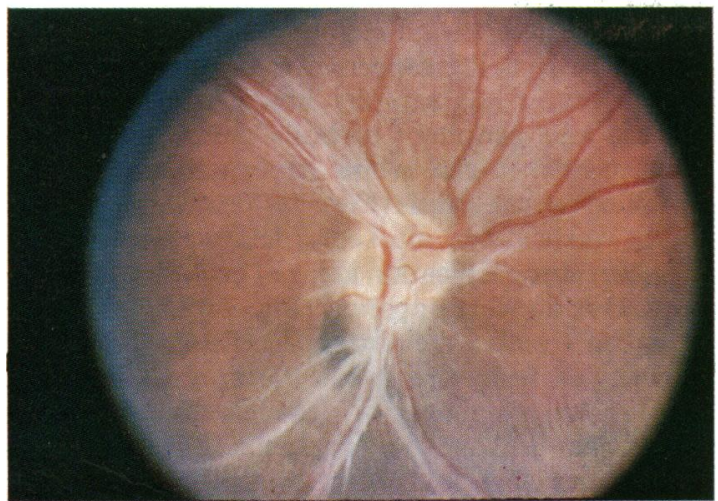

Fig. 1 Posterior pole, right eye, Case 1, shows markedly sheathed retinal vessels. Arteries are more involved than veins

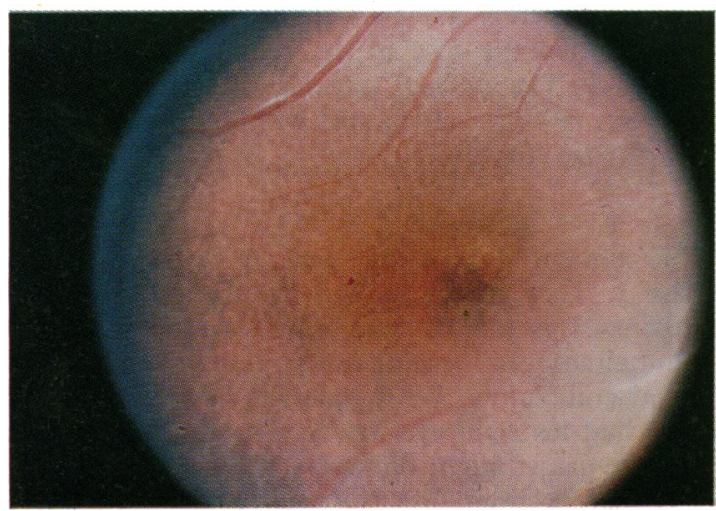

Fig. 3 Right eye, Case 1. Foveal area shows confluence of many cystoid spaces. Remainder of posterior pole shows reticular pattern that is most marked temporal to macula

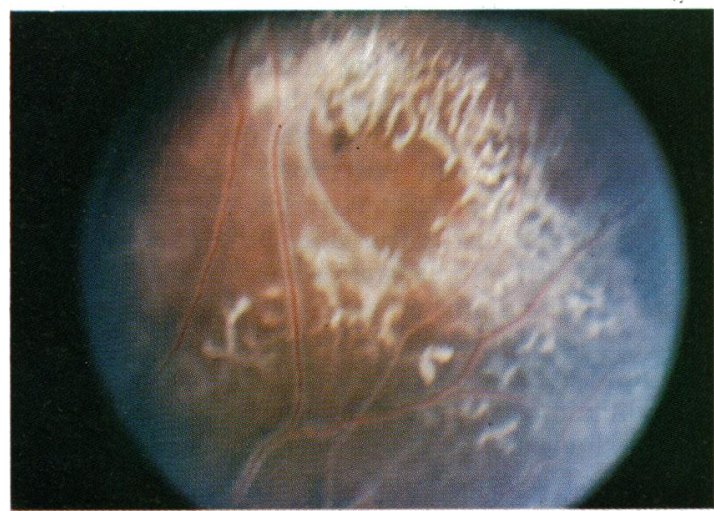

Fig. 5 Left eye, Case 1. Large inner layer retinal hole is visible in area of schisis. Opacified retinal vessels can be identified in both inner and outer retinal layers

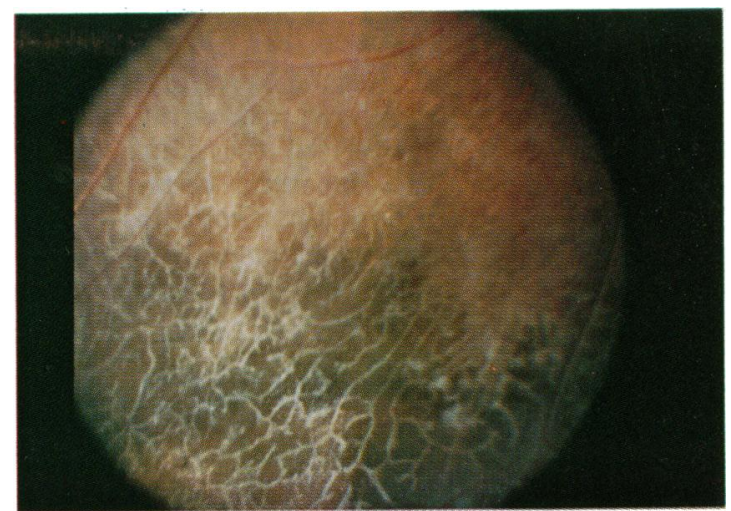

Fig. 2 Right eye, Case 1, showing inferotemporal retina. Large raised schisis cavity is seen with opacified retinal vessels and pigment clumping

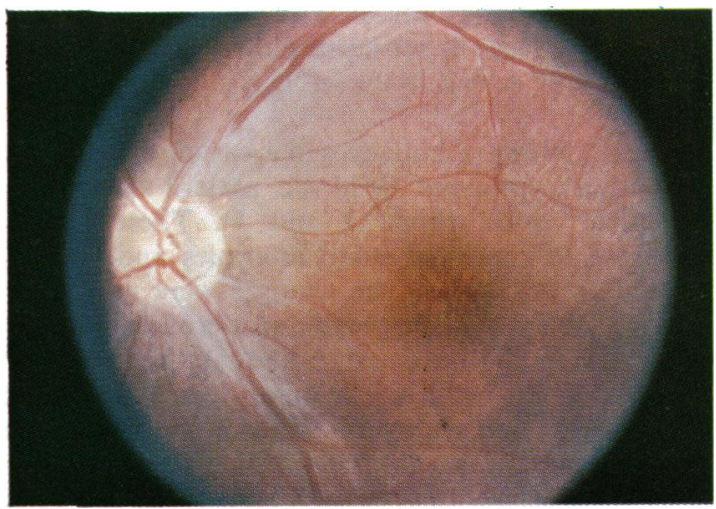

Fig. 4 Left eye, Case 1, showing classic bicycle-spoke pattern of cystoid spaces in macula. Reticular pattern seen in right eye is absent

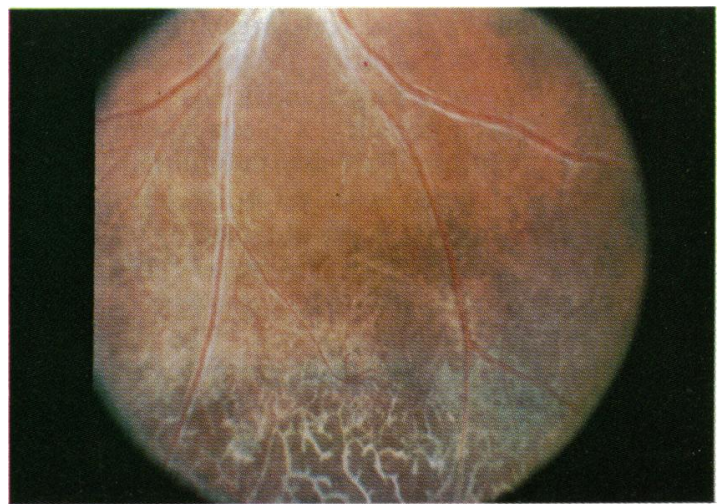

Fig. 6 Right eye, Case 1, shows extension of vascular opacification into flat apparently nonschitic retina 


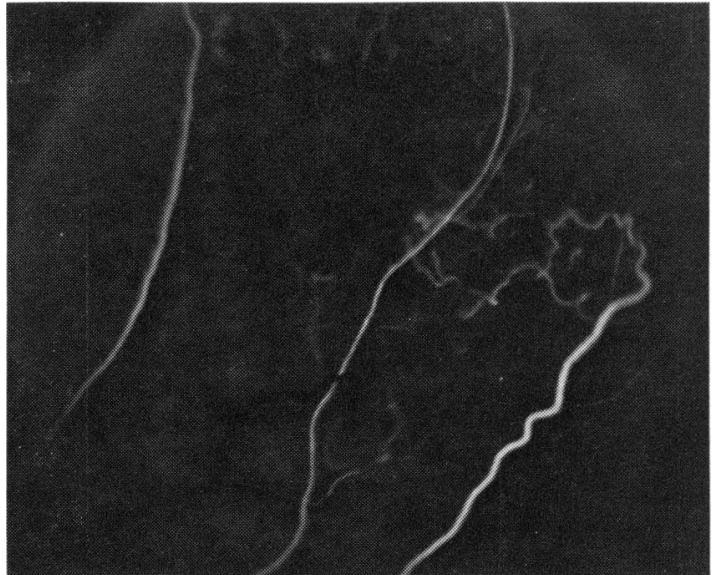

Fig. 7a Sequence of filling of retinal vessels in area of schisis. Arterial phase shows very slow filling of vessels

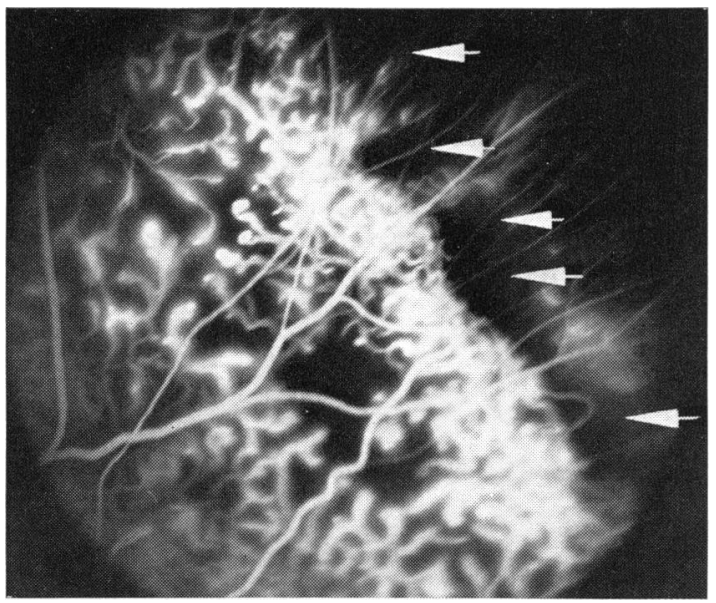

Fig. 7c Slightly more anterior view shows extensive capillary dropout (arrows) anterior to opacified vessels. Staining of opacified vessel walls is apparent with some intraretinal leakage of fluorescein

remnant of the inner layer of schisis was present in the vitreous.

Fluorescein angiography showed no macular leakage in either eye but intraretinal leakage and capillary nonperfusion temporally in the left eye in the area of schisis. The ERG demonstrated selective depression of the b-wave under photopic and scotopic conditions. The scotopic changes were more pronounced, however.

\section{Discussion}

Congenital retinoschisis was first described concurrently in 1932 by Thomson (1932) and Anderson

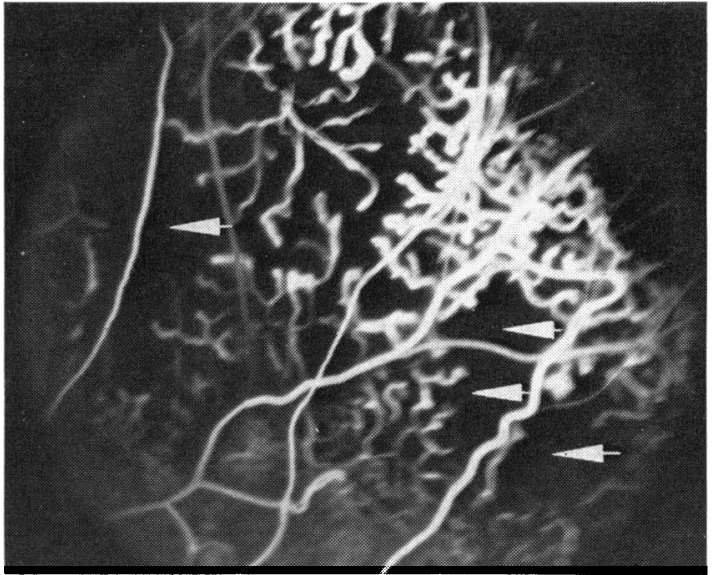

Fig. $7 \mathrm{~b}$ Venous phase shows that arborescent white structures seen in Fig. 5 are retinal vessels. Some areas of capillary dropout are present (arrows)

(1932). Thomson's report included a description of the characteristic radial spokes in the macula as well as peripheral pigment clumping and retinal elevation. Jager (1953) published the first account in which splitting of the inner retinal layers was identified as the origin of the vascular veils in the vitreous. He termed this process retinoschisis. Subsequent histopathological studies (Yanoff et al., 1968; Manschot, 1972) have suggested that the splitting occurs mainly in the nerve fibre layer.

Fluorescein angiography has been used to study the macula in X-linked retinoschisis. These studies have in general shown no leakage of fluorescein into

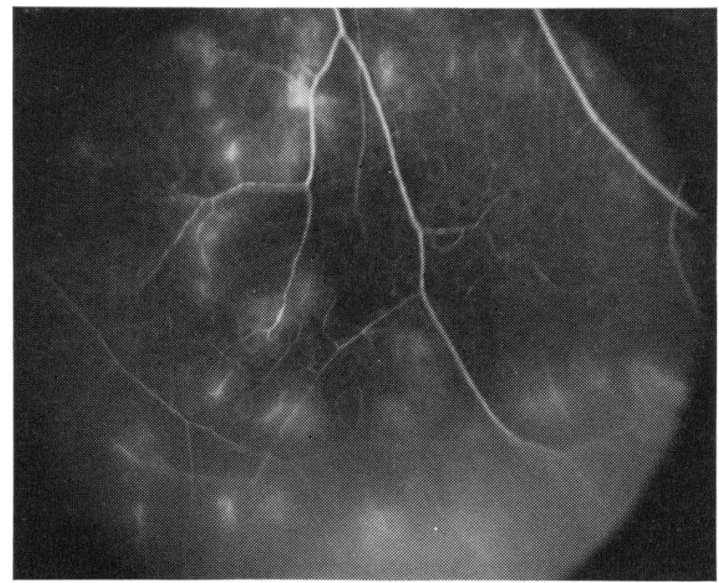

Fig. 8 Late phase angiogram in right eye shows patchy vessel wall staining and leakage at margin of schisis in clinically nonschitic retina (above) as well as schitic retina (below) 


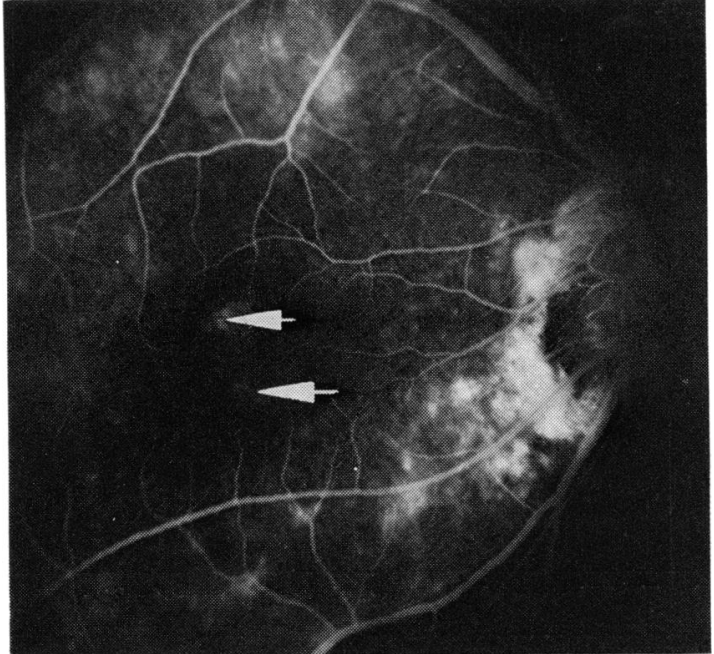

Fig. 9a Posterior pole, right eye, Case 1. Angiogram shows pigment disruption under inferotemporal vessels with 2 small areas of pigment irregularity under macula (arrows). No leakage into cystoid spaces is present

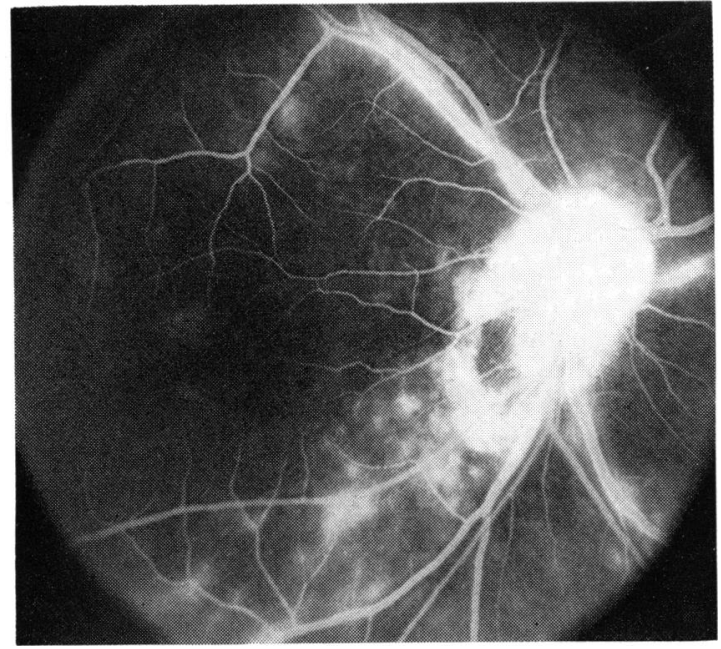

Fig. 9b Late phase angiogram shows prominent staining of disc and large vessels, especially veins

the cystoid spaces in the macula (above). However, in some cases faint pooling of fluorescein has been found (Ewing and Cullen, 1972). Both our cases showed classic bicycle-spoke patterns in the macula with no fluorescein leakage. In both eyes of Case 1 there was prominent staining of the optic disc and large vessel walls with some intraretinal leakage of dye in the posterior pole. Disruption of the retinal pigment epithelium under the macula and near the disc was present in the right eye only.
Previous authors have commented briefly on fluorescein angiography of the periphery of patients with X-linked retinoschisis. Tasman (1975) noted pigment epithelial atrophy in the areas underlying a vitreous veil. Ewing and Ives (1969) and Ewing and Cullen (1972) mentioned that the dendritic structures in the periphery were patent vessels that fill with fluorescein. They noted delayed circulation in many cases and suggested that the vessels may in some instances be neovascular (Ewing and Ives, 1969) and that the fundus picture in X-linked retinoschisis could resemble Eales's disease. The first case presented here shows vividly that these white dendritic structures are opacified vessels. In areas of advanced schisis a few of the dendritic vessels were not perfused. Elsewhere a slow filling of opacified vessels was noted. No neovascularisation was seen. Presumably vessels in both the deep and superficial capillary networks of the retina were opacified, as in some areas dendritic structures were visible in both the inner and outer layers of the schisis cavity. At the anterior margins of the schisis large areas of capillary nonperfusion were present. The retinal capillaries in these areas seemed to have totally disappeared. At the posterior margins of the schisis early vessel opacification and some fluorescein leakage were noted; these changes extended into flat nonschitic retina. Case 2 also showed opacified

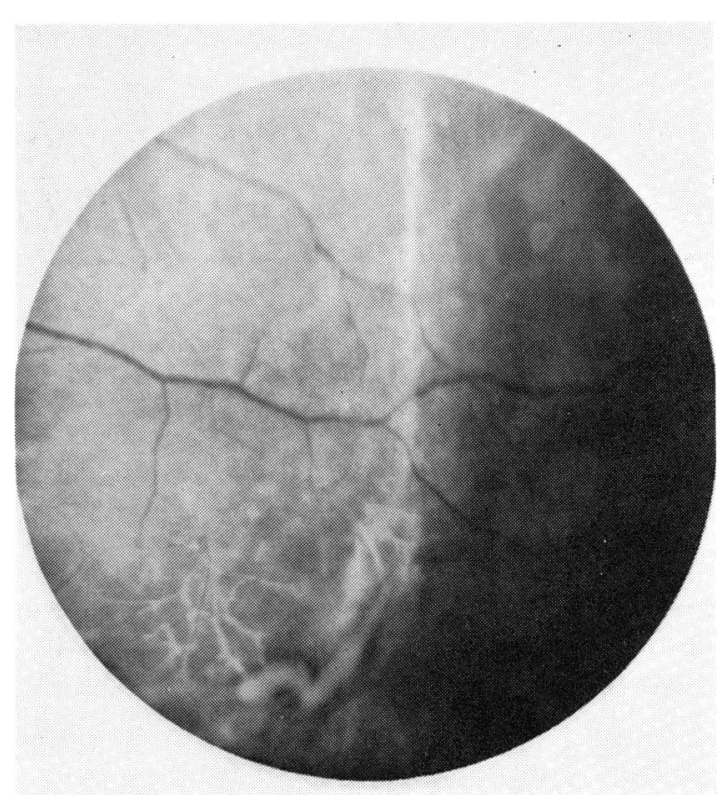

Fig. 10 Temporal periphery, left eye, Case 2, shows ridge of retinoschisis with early vascular opacification along inferior margin of ridge 
vessels. However, the changes were subtle and could have been overlooked without careful examination of the retinal periphery. This case also showed the characteristic depression of the b-wave of the ERG, which can be seen with even minimal peripheral retinoschisis.

Retinal vascular leakage and opacification have also been described in association with the FavreGoldmann syndrome (Fishman et al., 1976). Although the Favre-Goldmann syndrome also shows retinoschisis, it is an autosomal recessive disease characterised by night blindness and photoreceptor degeneration. The presence of vascular leakage and opacification in both these hereditary diseases with retinoschisis is puzzling. It is possible that abnormalities of the retinal circulation could contribute to the development of the retinoschisis. The fact that these areas of retinoschisis are not totally avascular does not detract from this hypothesis. The decreased blood flow in these areas could result in localised ischaemia that might contribute to the resultant separation of the inner from the outer retinal layers. Retinal vascular leakage could also play a role in schisis formation. Conversely, it is also possible that the vascular opacification and leakage are secondary to the retinoschisis, though we believe that this is unlikely, since vascular sheathing and early vessel wall opacification and leakage were seen in our patients and in some patients with Favre-Goldmann syndrome (Fishman et al., 1976) even in areas where no schisis was present. The origin of the vascular changes in these two diseases remains uncertain.

Differentiation of X-linked retinoschisis from the much rarer Favre-Goldmann syndrome is not difficult. Our patients complained of no night blindness (which is an early symptom in the FavreGoldmann syndrome), and the dark adaptation curve in Case 1 showed only minimal increase of rod final thresholds. In addition the maculae of our patients had the bicycle-spoke pattern characteristic of X-linked juvenile retinoschisis. The macular changes in the Favre-Goldmann syndrome are much more subtle (Fishman et al., 1976). In addition, ERG findings in the Favre-Goldmann syndrome (Fishman et al., 1976) show abnormalities of both the a- and b-waves, often with nondetectable amplitudes. In X-linked retinoschisis ERG abnormalities are limited to depression of the b-wave except in markedly advanced stages. Macular changes similar to $\mathrm{X}$-linked retinoschisis have also recently been reported in a family with familial foveal retinoschisis (Lewis et al., 1977). These patients, however, had normal ERG recordings.

Dr Gerald Fishman performed the electrophysiological measurements, and Mr Norbert Jednock, OP, assisted with the photography.

\section{References}

Anderson, J. R. (1932). Anterior dialysis of retina: disinsertion or avulsion at ora serrata. British Journal of Ophthalmology, 16, 705-727.

Burns, R. P., Lovrien, W. E., and Cibis, A. B. (1971). Juvenile sex-linked retinoschisis: clinical and genetic studies. Transactions of the American Academy of Ophthalmology and Otolaryngology, 75, 1011-1021.

Cibis, P. A. (1965). Retinoschisis-retinal cysts. Transactions of the American Ophthalmological Society, 63, 417-453.

Constantaras, A. A., Dobbie, J. G., Choromokos, E. A., and Frenkel, M. (1972). Juvenile sex-linked retinoschisis in a black family. American Journal of Ophthalmology, 74, 1166-1178.

Deutman, A. F. (1977). Vitreoretinal dystrophies. In Hereditary Retinal and Choroidal Diseases, Vol. 2, pp. 1043-1062. Edited by A. E. Krill and D. B. Archer. Harper \& Row: Hagerstown, Maryland.

Ewing, C. C., and Cullen, A. P. (1972). Fluorescein angiography in X-chromosomal maculopathy with retinoschisis (juvenile hereditary retinoschisis). Canadian Journal of Ophthalmology, 7, 19-28.

Ewing, C. C., and Ives, E. J. (1969). Juvenile hereditary retinoschisis. Transactions of the Ophthalmological Societies of the United Kingdom, 89, 29-39.

Fishman, G. A., Fishman, M., and Maggiano, J. (1977). Macular lesions associated with retinitis pigmentosa. Archives of Ophthalmology, 95, 798-803.

Fishman, G. A., Jampol, L. M., and Goldberg, M. F. (1976). Diagnostic features of the Favre-Goldmann syndrome. British Journal of Ophthalmology, 60, 345-353.

Harris, G. S., and Yeung, J. W. (1976). Maculopathy of sexlinked juvenile retinoschisis. Canadian Journal of Ophthalmology, 11, 1-10.

Helve, J. (1972). Colour vision in X-chromosomal juvenile retinoschisis. Modern Problems in Ophthalmology, 11, 122-129.

Jager, G. M. (1953). A hereditary retinal disease. Transactions of the Ophthalmological Societies of the United Kingdom, 73, 617-619.

Krause, U., Vainio-Mattila, B., Eriksson, A., and Forsius, H. (1970). Fluorescein angiographic studies on X-chromosomal retinoschisis. Acta Ophthalmologica, 48, 794-807.

Lewis, R. A., Lee, B. G., Martonyi, C. L., Barnett, J. M., and Falls, H. F. (1977). Familial foveal retinoschisis. Archives of Ophthalmology, 95, 1190-1196.

Manschot, W. A. (1972). Pathology of hereditary juvenile retinoschisis. Archives of Ophthalmology, 88, 131-138.

Tasman, W. (1975). Macular changes in congenital retinoschisis. Modern Problems in Ophthalmology, 15, 40-47.

Thomson, E. (1932). Memorandum regarding a family in which neuroretinal disease of an unusual kind occurred only in males. British Journal of Ophthalmology, 16, 681-686.

Yanoff, M., Rahn, E. K., and Zimmerman, L. E. (1968). Histopathology of juvenile retinoschisis. Archives of Ophthalmology, 79, 49-53. 